\title{
Genetic engineering approaches for the fermentative production of phenylglycines
}

\author{
David Moosmann ${ }^{1} \cdot$ Vladislav Mokeev $^{1}$ - Andreas Kulik ${ }^{1}$ - Natalie Osipenkov ${ }^{1}$. Susann Kocadinc ${ }^{1}$. \\ Regina Ort-Winklbauer ${ }^{1}$. Franziska Handel ${ }^{1}$. Oliver Hennrich ${ }^{1} \cdot$ Jung-Won Youn ${ }^{2} \cdot$ Georg A. Sprenger $^{2}$. \\ Yvonne Mast ${ }^{1,3,4,5}$ iD
}

Received: 16 November 2019 / Revised: 29 January 2020 / Accepted: 6 February 2020 / Published online: 20 February 2020

(C) The Author(s) 2020

\begin{abstract}
L-phenylglycine (L-Phg) is a rare non-proteinogenic amino acid, which only occurs in some natural compounds, such as the streptogramin antibiotics pristinamycin I and virginiamycin S or the bicyclic peptide antibiotic dityromycin. Industrially, more interesting than L-Phg is the enantiomeric D-Phg as it plays an important role in the fine chemical industry, where it is used as a precursor for the production of semisynthetic $\beta$-lactam antibiotics. Based on the natural LPhg operon from Streptomyces pristinaespiralis and the stereo-inverting aminotransferase gene hpgAT from Pseudomonas putida, an artificial D-Phg operon was constructed. The natural L-Phg operon, as well as the artificial D-Phg operon, was heterologously expressed in different actinomycetal host strains, which led to the successful production of Phg. By rational genetic engineering of the optimal producer strains $S$. pristinaespiralis and Streptomyces lividans, Phg production could be improved significantly. Here, we report on the development of a synthetic biology-derived D-Phg pathway and the optimization of fermentative Phg production in actinomycetes by genetic engineering approaches. Our data illustrate a promising alternative for the production of Phgs.
\end{abstract}

Keywords Synthetic biology · Genetic engineering $\cdot$ Non-proteinogenic amino acids $\cdot$ D-amino acids $\cdot$ Phenylglycine Actinomycetes

Electronic supplementary material The online version of this article (https://doi.org/10.1007/s00253-020-10447-9) contains supplementary material, which is available to authorized users.

Yvonne Mast

yvonne.mast@dsmz.de

1 Microbiology/Biotechnology, Interfaculty Institute of Microbiology and Infection Medicine, Faculty of Science, University of Tübingen, Auf der Morgenstelle 28, D-72076 Tübingen, Germany

2 Institute of Microbiology, University Stuttgart, Allmandring 31, D-70569 Stuttgart, Germany

3 German Center for Infection Research (DZIF), Partner Site Tübingen, Tübingen, Germany

4 Department "Bioresources for Bioeconomy and Health Research", Leibniz Institute DSMZ-German Culture Collection for Microorganisms and Cell Cultures, 38124 Braunschweig, Germany

5 Institute for Microbiology, Technical University of Braunschweig, 38106 Braunschweig, Germany

\section{Introduction}

To date, more than 900 naturally occurring amino acids have been identified ( $\mathrm{Lu}$ and Freeland 2006) of which the 20 proteinogenic L-amino acids only constitute $2 \%$. The majority of the residual $98 \%$ of non-proteinogenic amino acids serve as building blocks for bioactive natural compounds (Walsh et al. 2013). Non-proteinogenic amino acids are becoming ever more important as tools for modern drug discovery and development. On the one hand, freestanding non-proteinogenic amino acids act as antimetabolites of common amino acids and are effective inhibitors for various metabolic targets. Besides that, non-proteinogenic amino acids serve as building blocks for numerous bioactive compounds and drugs. Especially D-amino acids are of particular importance for the production of pharmaceuticals and fine chemicals. They are utilized in drugs, drug intermediates, food additives, artificial sweeteners, deodorants, insecticides, or commodity chemicals (Barredo 2005; Global Industry Analysts Inc. 2016). Annually, several tons of D-amino acids are produced. 
Due to an aging world population, there is a strong demand for dietary and pharmaceutical supplements, which in turn will increase the need for D-amino acids in the coming years (Global Industry Analysts Inc. 2016). One of the industrially relevant D-amino acids is D-phenylglycine (D-Phg), which is used as precursor for the production of various $\beta$-lactam antibiotics. D-Phg is a constituent of a number of semisynthetic penicillins (ampicillin, apalcillin (Boehringer Ingelheim), mezlocillin (Bayer), pivampicillin, LEO Pharma), etc.) and cephalosporins (cefalexin, cefradine, cefaclor, cefaloglycine, etc.) (Stevenazzi et al. 2014; Shiau et al. 2005; Schmid et al. 2001; Müller et al. 2013). Currently, D-Phg is produced in a scale $>5000$ tons per year worldwide (Vedha-Peters et al. 2006). Until now, the amino acid is synthesized by classical or enzymatic resolution of a racemic mixture (Wegman et al. 2001). This production process is based on petrochemical feedstocks. The disadvantage of such a conventional method is that it includes many individual processing steps, which make the entire production process commercially less attractive. Besides that, chemical syntheses often need numerous chemicals and solvents, are energetically unfavorable, and/or produce a lot of waste substances. It would therefore be highly desirable to avail a more environmentally friendly fermentative route for the production of the unnatural amino acid D-Phg in future. With a fermentative production process, the substance of interest is produced by microorganisms obtained from renewable raw materials, such as glucose, whereby the end-products are characterized by a high chemo-, regio-, and stereo-selectivity. In terms of Phg, fermentative production was hampered by the fact that the amino acid is not accessible by any natural biosynthetic pathway from microbes or other organisms. In a previous approach, an artificial D-Phg production pathway has been designed in $E$. coli that applies three different enzymes from three different organisms (HmaS-hydroxymandelate synthase from Amycolatopsis orientalis, Hmo-hydroxymandelate oxidase from Streptomyces coelicolor and HpgAT-D-(4hydroxy)phenylglycine aminotransferase from Pseudomonas putida), which led to the successful production of D-Phg (Müller et al. 2006). Only recently, the first natural Phg biosynthetic pathway has been reported for the antibiotic producer Streptomyces pristinaespiralis (Mast et al. 2011a; Osipenkov et al. 2018), which can now serve as the basis for the development of a fermentative D-Phg production route.

S. pristinaespiralis is the producer of the streptogramin antibiotic pristinamycin, which consists of the two chemically non-related substances pristinamycin I (PI) and pristinamycin II (PII). PI is synthesized by the nonribosomal peptide synthetases (NRPSs) SnbA, SnbC, and SnbDE, whereby the latter one incorporates L-Phg as the final amino acid into the growing PI peptide chain (Mast et al. 2011b; Mast and Wohlleben 2014). Within the pristinamycin biosynthetic gene region, the genes $p g l A, p g l B, p g l C, p g l D$, and $p g l E$ are organized in an operon-like structure (lpg) and together encode for L-Phg biosynthesis (Mast et al. 2011a, 2015, Osipenkov 2016). These genes are located downstream of the NRPS genes $s n b C$ and $s n b D E$ and are under control of the pathway-specific transcriptional activator PapR2 (Mast et al. 2015) (Fig. 1a).

L-Phg in S. pristinaespiralis is suggested to originate from the shikimate pathway. As a first metabolic step, phenylpyruvate is converted to phenylacetyl-CoA by the action of a pyruvate dehydrogenase-like complex PglB/C. Phenylacetyl-CoA is oxidized to benzoylformyl-CoA via the Phg dioxygenase PglA. The CoA residue from benzoylformyl-CoA is cleaved off by the thioesterase PglD, resulting in the formation of phenylglyoxylate. In a final reaction step, phenylglyoxylate is converted to L-Phg by the aminotransferase PglE (Mast et al. 2011b). As PglE uses L-phenylalanine as amino group donor for the transamination reaction, phenylpyruvate is formed as the $\alpha$ keto acid product, which can re-enter Phg biosynthesis as a precursor (Osipenkov et al. 2018) (see Fig. 2).

L-Phg is a rare amino acid, which only occurs in a few natural products, such as the related streptogramin antibiotic virginiamycin S from Streptomyces virginiae (Ningsih et al. 2011) or the bicyclic peptide antibiotics dityromycin, produced by Streptomyces sp. strain AM-2504; GE82832 of Streptosporangium cinnabarinum strain GE82832, or MBJ0086 and MBJ-0087, isolated from Sphaerisporangium sp. 3226 (Al Toma et al. 2015). There is also an industrial demand for L-Phg since it is used as a component of the synthetic cyclic hexadepsipeptide pasireotide (Signifor ${ }^{\circledR}$, Novartis), which is a somatostatin analogue used for the treatment of Cushing's disease. L-Phg is also used for the synthesis of the antitumor compound taxol (Croteau et al. 2006; Denis et al. 1991; Wang et al. 1994) and the synthesis of DAPT (N-[N-(3,5-difluorophenacetyl)-L-alanyl]-S-phenylglycine tbutyl ester), which acts as an inhibitor of the human $\gamma$ secretase, a target used for the treatment of Alzheimer's disease and different types of cancer (Kan et al. 2004). Besides its application for diverse pharmaceuticals, L-Phg, as well as the enantiomeric D-Phg, can be used for the synthesis of the artificial non-nutritive sweetener aspartame (Ebeling 1998; Janusz 1986; Schutt 1981).

In this study, we describe the development of a synthetic biology-derived D-Phg pathway. Furthermore, we report on genetic engineering approaches in order to optimize Phg production in actinomycetal expression strains.

\section{Material and methods}

\section{Bacterial strains, plasmids, and cultivation conditions}

Bacterial strains, plasmids, cosmids, and primers used in this study are listed in Table S1. An overview of genes used for this study is given in Table 1. For routine cloning strategies, Escherichia coli XL1-Blue was used. E. coli strains were grown 
a)

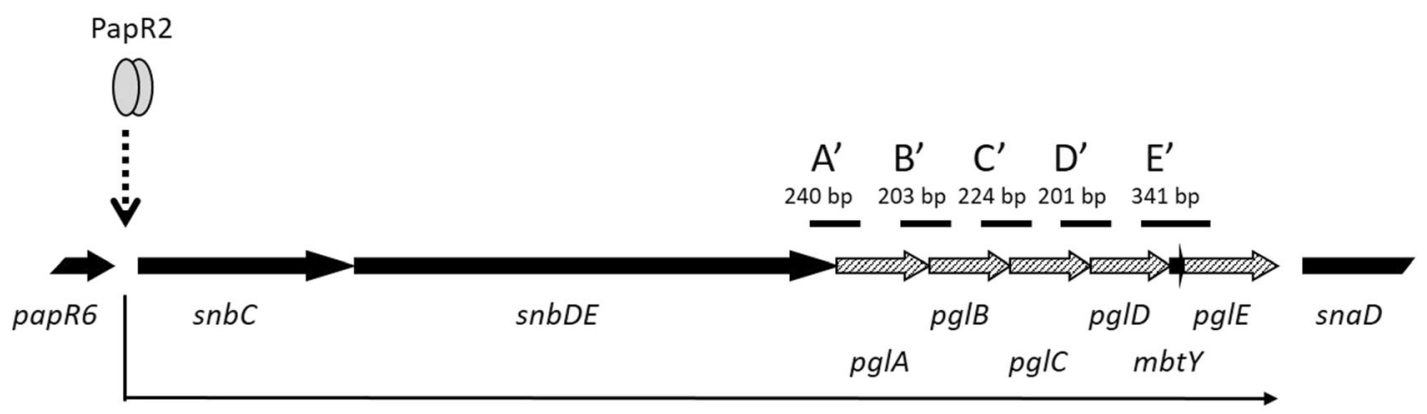

b)

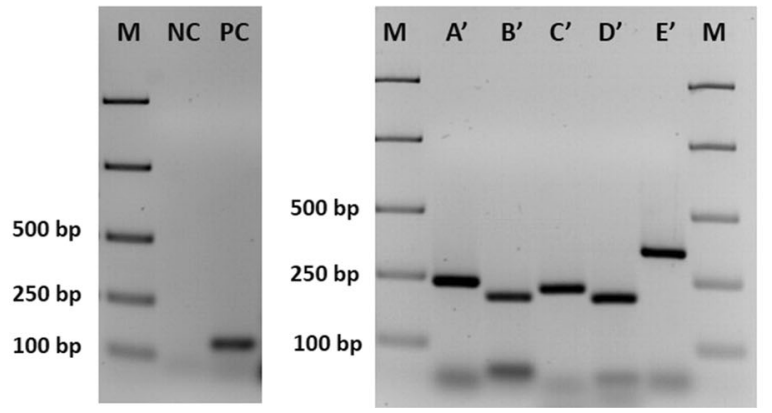

Fig. 1 Schematic presentation of the lpg operon. L-Phg biosynthesis genes are shown as dashed arrows and adjacent genes as black arrows. Predicted RT-PCR amplificates $\left(\mathrm{A}^{\prime}-\mathrm{E}^{\prime}\right)$ are shown as black lines. Transcriptional activation by PapR2 (gray ellipses) is indicated as broken arrow. Thin black arrow depicts co-transcription of genes (a). Transcriptional analysis of the lpg operon in S. pristinaespiralis. Total

in Luria-Bertani (LB) medium at $37{ }^{\circ} \mathrm{C}$ (Sambrook et al. 1989) supplemented with kanamycin or apramycin $(50$ or $100 \mu \mathrm{g} / \mathrm{ml}$, respectively) when appropriate. For cultivation and harvesting of genomic DNA, Streptomyces strains were grown in $100 \mathrm{ml}$ of
RNA was harvested after $24 \mathrm{~h}$. Left figure shows RT-PCR results with $h r d B$-specific primers and total RNA (negative control, NC) and cDNA (positive control, PC) as template, respectively. Right figure shows RTPCR results from amplification of $p g l$ gene overlapping regions (amplificate $\mathrm{A}^{\prime}, \mathrm{B}^{\prime}, \mathrm{C}^{\prime}, \mathrm{D}^{\prime}$, and $\mathrm{E}^{\prime}$, respectively). A total of $5 \mu \mathrm{l}$ of the $1 \mathrm{~kb}$ ladder from Fermentas was used as an internal standard (M) (b)

S-medium (Kieser et al. 2000) in 500-ml Erlenmeyer flasks (with steel springs) on an orbital shaker $(180 \mathrm{rpm})$ at $28^{\circ} \mathrm{C}$. For pristinamycin production analyses, cells were grown and treated as reported previously (Mast et al. 2011a).

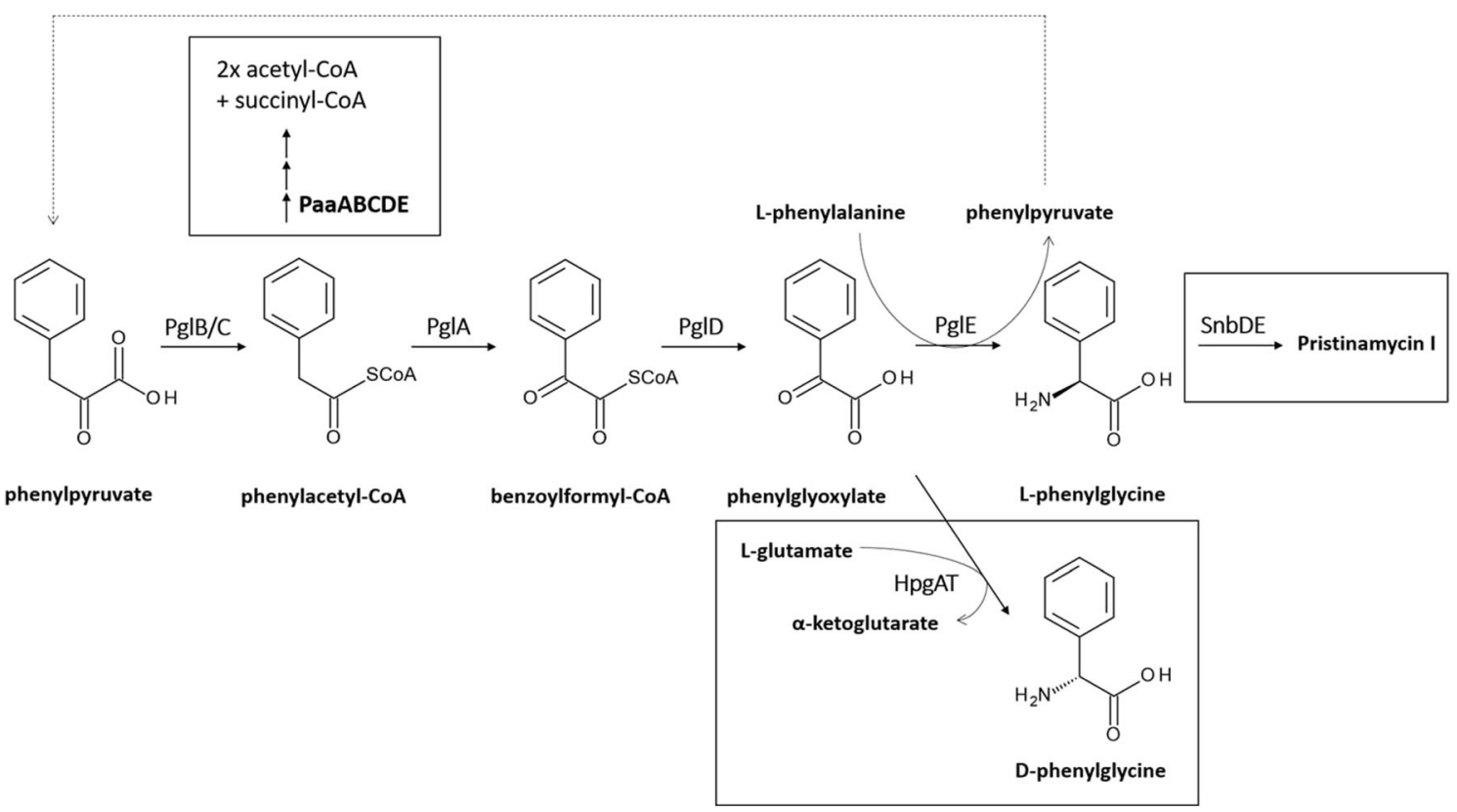

Fig. 2 Schematic presentation of the natural L-Phg biosynthetic pathway from $S$. pristinaespiralis. Biochemical reactions targeted by genetic engineering, such as the HpgAT catalyzed reaction resulting in D-Phg production, PaAABCDE-catalyzed Phenylacetyl-CoA degradation, and SnbDE-catalyzed incorporation of L-Phg into PI, are highlighted in black boxes 
Table 1 List of genes mentioned in this study with encoded functions

\begin{tabular}{lll}
\hline Gene & Origin & Function \\
\hline$p g l A$ & S. pristinaespiralis & Phenylglycine dehydrogenase \\
$p g l B$ & S. pristinaespiralis & Pyruvate dehydrogenase $\alpha$-subunit \\
$p g l C$ & S. pristinaespiralis & Pyruvate dehydrogenase $\beta$-subunit \\
$p g l D$ & S. pristinaespiralis & Thioesterase \\
pglE & S. pristinaespiralis & L-phenylglycine aminotransferase \\
hpgAT & P. putida & D-phenylglycine aminotransferase \\
$\operatorname{snbDE}$ & S. pristinaespiralis & Pristinamycin I-specific nonribosomal peptide \\
& & synthetase \\
papR5 & S. pristinaespiralis & TetR-like repressor of pristinamycin biosynthesis \\
paaABCDE (paa operon) & S. pristinaespiralis, & phenylacetyl-CoA epoxidase multicomponent \\
& S. lividans & enzyme system \\
\hline
\end{tabular}

\section{Transcriptional analysis by RT-PCR experiments}

S. pristinaespiralis $\operatorname{Pr} 11$ wild type was grown in pristinamycin inoculum and production medium as reported previously (Mast et al. 2011a). Samples were harvested after $24 \mathrm{~h}$. RNA isolation and RT-PCR procedure were carried out as described before (Mast et al. 2015). For RT-PCR reactions, primers RTpglfw/rv were used that anneal to overlapping regions of the $p g l$ gene sequences. As an internal control, RTPCR was performed with primers targeting the major sigma factor transcript $h r d B$. To exclude DNA contamination, negative controls were carried out by using total RNA as a template for each RT-PCR reaction.

\section{Construction of Phg expression plasmids}

\section{Ipg expression construct}

For cloning of the native lpg operon from S. pristinaespiralis, the pYJM1 cosmid DNA, harboring the pglA-E genes, was used as a template in a PCR approach with the primers lpgfw/ rv and KAPAHiFi ${ }^{\mathrm{TM}}$ polymerase (Peqlab). The lpgfw/rv primer pair was designed in a way that a NdeI ( $5^{\prime}$ end) and HindIII ( $3^{\prime}$ end) restriction sequence is added to the lpg amplificate. The $\sim 6-\mathrm{kb}$ lpg fragment was subcloned into the linear PCR cloning vector pJET1.2/blunt (Fermentas), which resulted in the construct pJET/lpg. lpg was isolated from $\mathrm{pJET/lpg}$ as a NdeI/HindIII fragment and was cloned into the NdeI/HindIIIrestriction site of the expression vector pRM4 under control of the constitutive erythromycin resistance gene promoter, $P_{\text {ermE }}$, resulting in the lpg expression construct pYM/lpg (Fig. S1).

\section{dpg expression construct}

On the basis of the natural lpg operon from S. pristinaespiralis, an artificial $d p g$ operon was constructed. For this purpose, the Ndel/HindIII lpg fragment from $\mathrm{pJET} / \mathrm{lpg}$ (see above) was subcloned into the NdeI/HindIII-restricted E. coli vector $\mathrm{pK} 18$
(Pridmore 1987), resulting in construct pK18/lpg. For cloning of the $d p g$ operon, a recombinant PCR approach was conducted in order to fuse the $p g l D$ gene from $S$. pristinaespiralis to the $h p g A T$ gene of $P$. putida. To amplify the $p g l D$ fragment, the pYJM1 cosmid DNA was used as a template together with primers pglDfus $1 / 2$ for PCR amplification, resulting in fragment $p g l D^{\prime}$ with a size of $\sim 800 \mathrm{bp}$. The $h p g A T$ gene (accession number AX467211) from P. putida was synthesized de novo (Mr. Gene GmbH, Regensburg) and used as a template for PCR amplification with primers hpgATfus $1 / 2$, which resulted in the $\sim 1.4$-kb fragment $h p g A T^{\prime}$. Primers pglDfus2 and hpgATfus1 had 20-bp complementary 5'-3' sequences, which allowed annealing of the fragments in a recombinant PCR approach. $p g l D^{\prime}$ and $h p g A T^{\prime}$ were used as templates for recombinant PCR with primers pglDfus1/hpgATfus2, resulting in the fusion product $p g l D-h p g A T^{\prime}(\sim 2.2 \mathrm{~kb})$. pglD-hpgAT' was subcloned in the PCR cloning plasmid pDrive (Qiagen), which resulted in the construct pDrive/pglD-hpgAT'. The correctness of the gene sequence was verified by the primer walking method (GATC Biotech, Konstanz). The plasmid pK18/lpg was cleaved with SfiI/HindIII and the $\sim 6.3-\mathrm{kb}$ pK18/pglA-pglC fragment was ligated to the $p g I D-h p g A T^{\prime}$ fragment, which was excised with the same restriction enzymes from pDrive/pglD- $h p g A T^{\prime}$, resulting in the construct $\mathrm{pK} 18 / \mathrm{dpg}$. The artificial $d p g$ operon was isolated from $\mathrm{pK} 18 / \mathrm{dpg}$ as a NdeI/HindIII fragment and was subcloned into the NdeI/HindIII-restricted pRM4 plasmid, resulting in the $d p g$ expression construct pYM/dpg (Fig. S1).

\section{Expression constructs with thiostrepton resistance cassettes}

In order to select for Phg operon containing transformants of apramycin-resistant mutants (MpglE (Mast et al. 2011a) and papR5::apra (Mast et al. 2015)), expression constructs were designed, which harbor a thiostrepton resistance cassette $\left(\right.$ thio $\left.^{R}\right)$. For this purpose, the thio ${ }^{R}$ cassette was isolated as a $X b a \mathrm{I}$-restricted fragment from pDrive-thio and was cloned into the $\mathrm{Xba \textrm {I }}$ restriction site of $\mathrm{pRM} 4, \mathrm{pYM} / \mathrm{lpg}$, and $\mathrm{pYM} /$ 
$\mathrm{dpg}$, resulting in the expression constructs pYMT, pYMT/lpg, and pYMT/dpg, respectively (Fig. S1).

\section{Transformation of strains and culture conditions}

The targeting plasmids $\mathrm{pYM} /$ lpg and $\mathrm{pYM} / d p g$ were each transferred to S. pristinaespiralis $\operatorname{Pr} 11, S$. lividans T7, $S$. albus, A. balhimycina, and $R$. jostii RHA1 by protoplast transformation (Kieser et al. 2000), resulting in the expression strains SPlpg-OE, SPdpg-OE, SLlpg-OE, SLdpg-OE, SAlpg$O E$, SAdpg-OE, ABlpg-OE, ABdpg-OE, RJlpg-OE, and $R J d p g-O E$, respectively. Strains with the empty pRM4 vector served as control $(S P-C, S L-C, S A-C, A B-C$, and $R J-C$, respectively). All strains were inoculated from R5 agar into three independent replicates of $100 \mathrm{ml}$ of preculture medium (R5 or pristinamycin production medium HT7T). R5 contained (per liter) the following: sucrose $103 \mathrm{~g}$; yeast extract, $5 \mathrm{~g}$; glucose, $10 \mathrm{~g}$; TES ([N-tris(hydroxymethyl)methyl-2aminoethanesulfonic acid], $5.75 \mathrm{~g} ; \mathrm{K}_{2} \mathrm{SO}_{4}, 0.25 \mathrm{~g} ; \mathrm{MgCl}_{2}$, $10.12 \mathrm{~g}$; casamino acids, $0.1 \mathrm{~g}$; L-proline, $3 \mathrm{~g}$; $\mathrm{KH}_{2} \mathrm{PO}_{4}$, $0.05 \mathrm{~g} ; \mathrm{CaCl}_{2} \times 2 \mathrm{H}_{2} \mathrm{O}, 2.94 \mathrm{~g}, 2 \mathrm{ml}$ of trace elements stock solution; pH 7.4 and HT7T contained (per liter): white dextrin, $10 \mathrm{~g}$; NZ amine-A, 2 g; LabLemco beef powder, $1 \mathrm{~g}$; yeast extract, $1 \mathrm{~g} ; 1 \mathrm{ml}$ of trace elements stock solution; $\mathrm{pH} 7.4$ (Kieser et al. 2000; Folcher et al. 2001). Strains were cultivated in 500-ml Erlenmeyer flasks (with steel springs) on an orbital shaker $(180 \mathrm{rpm})$ at $30^{\circ} \mathrm{C}$. After $72 \mathrm{~h}, 7 \mathrm{ml}$ of preculture were inoculated into $100 \mathrm{ml}$ of production medium (R5 or HT7T, respectively) and the main culture was grown for $24,30,48,72$, or $96 \mathrm{~h}$, respectively. Ten milliliters of sample was harvested and centrifuged at $5000 \mathrm{rpm}$ for $10 \mathrm{~min}$. One milliliter of culture filtrate was used for HPLCMS/MS analysis.

\section{Construction of mutants and mutant-derived expression strains}

Construction and verification of the mutants MsnbDE::thio, SPpaa::thio, and SLpaa::thio, as well as construction of all mutant-derived expression strains, is described in Supplementary File.

\section{HPLC-MS/MS analysis of phenylglycine}

HPLC-MS/MS analysis has been performed as described previously (Osipenkov et al. 2018). Tandem MS (MS/MS) was carried out in the positive mode for phenylglycine (Phg) (precursor ion $\mathrm{m} / \mathrm{z}$ 152) with the corresponding target mass. Phg amount was measured in counts corresponding to the peak height. Phg concentration was calculated by reference to a standard curve using suitable concentrations of pure Phg (Fluka). Data are presented as the averages of the results from three independent biological replicates.

\section{Results}

\section{Phg biosynthetic genes are co-transcribed as a multi-gene operon}

As described above, the L-Phg biosynthetic genes (pglA-E) are organized in an operon-like structure $(l p g)$ within the pristinamycin biosynthetic gene region (Fig. 1a) (Mast et al. 2011b). lpg is localized between the genes $s n b D E$ and $s n a D$, which encode PI- and PII-specific peptide synthetases, respectively (Mast et al. 2011a). The gene $m b t Y$ is embedded in the $l p g$ region and encodes a MbtH-like protein, which is suggested to interact with SnbDE but is not directly involved in Phg biosynthesis (Mast et al. 2011b). In order to determine if the $p g l$ genes are co-transcribed and to ensure a successful transcription of the lpg operon in the heterologous expression studies later on, RTPCR experiments have been conducted with RNA isolated from the $S$. pristinaespiralis wild type and primers that anneal to overlapping regions of the $p g l$ genes (Fig. 1a). With these experiments, amplicons were obtained, which are specific for the overlapping regions between $s n b D E$ and $p g l A\left(\mathrm{~A}^{\prime}\right), p g l A$ and $p g l B\left(\mathrm{~B}^{\prime}\right.$ ), $p g l B$ and $p g l C\left(\mathrm{C}^{\prime}\right), p g l C$ and $p g l D\left(\mathrm{D}^{\prime}\right)$, and $p g l D$ and $p g l E\left(\mathrm{E}^{\prime}\right.$ ), respectively, revealing that all $p g l$ genes are transcribed as one polycistronic mRNA and form an operon together with the Phgspecific NRPS gene $s n b D E$ (Fig. 1b). Since $s n b D E$ is located directly downstream of $s n b C$ with overlapping stop and start codons, respectively, and $s n b C$ has been shown to be regulated by PapR2, it can be estimated that $s n b C, \operatorname{snbDE}$, and the $p g l$ genes together form a multi-gene operon, which is under regulatory control of the pristinamycin pathway-specific activator PapR2 (Fig. 1a).

\section{Expression of L- and D-Phg operons in suitable host strains}

To obtain constructs for the fermentative production of L-Phg, the native $\sim 6-\mathrm{kb} \operatorname{lpg}$ operon from S. pristinaespiralis was cloned into the integrative vector pRM4 under control of the constitutive ermE* promoter, resulting in the expression construct $\mathrm{pYM} / \mathrm{lpg}$ (Fig. S1). For production of the D-Phg enantiomer, an artificial D-Phg operon $(d p g)$ was generated on the basis of the native lpg operon from S. pristinaespiralis: In a synthetic biology approach, the gene $p g l E$, encoding the L-Phg aminotransferase in $S$. pristinaespiralis, was exchanged by the gene hpgAT from P. putida, which codes for a stereospecific D-Phg aminotransferase. This D-Phg aminotransferase is the only currently known L to D stereo-inverting aminotransferase (Walton et al. 2018). A recombinant PCR yielded the artificial $d p g$ operon, which was cloned into pRM4, resulting in the expression construct $\mathrm{pYM} /$ dpg (Fig. S1). Both plasmids, pYM/lpg and pYM/dpg, were each transferred into different actinomycetes (S. pristinaespiralis $\operatorname{Pr} 11$, Streptomyces lividans T7, Streptomyces albus J1074, Amycolatopsis balhimycina, and Rhodococcus jostii RHA1) as 
homologous or heterologous host strains, respectively ( $-\mathrm{OE}$ strains; Supplementary File). Strains with the empty pRM4 vector served as control ( $-\mathrm{C}$ strains; Supplementary File). $S$. pristinaespiralis was used as expression strain because it is the natural producer of L-Phg, which is a building block for the biosynthesis of the streptogramin antibiotic PI. A. balhimycina was tested since it produces the structurally related nonproteinogenic amino acids hydroxy- and dihydroxyphenylglycine, which are components of the glycopeptide antibiotic balhimycin (Pfeifer et al. 2001). S. lividans and S. albus are established heterologous expression strains (Nah et al. 2017) and $R$. jostii has a well-studied, intensive aromatic compound metabolism (Yam et al. 2011). All strains were grown in R5 medium in triplicate. After 30-h, supernatant samples were harvested and Phg amount (given in $\mu \mathrm{g} / \mathrm{L}$ ) was determined by HPLC-MS/ MS analysis. Here, it should be noted that the applied method does not allow to distinguish between different Phg enantiomers. In order to determine enantiomerism of the produced Phg compounds, chiral HPLC analyses have been performed with various expression samples. However, Phg concentrations were too low to be detected (data not shown). HPLC-MS/MS analysis revealed that Phg amount was largest in samples from S. lividans (SL) and S. pristinaespiralis (SP) expression strains $(>1 \mu \mathrm{g} / \mathrm{L})$, whereas only minor Phg amounts were measured for samples of $S$. albus (SA), A. balhimycina (AB), and $R$. jostii (RJ) expression strains $(<0.75 \mu \mathrm{g} / \mathrm{L})$ (Fig. 3). No, or only trace amounts of Phg were detected in the respective pRM4 control samples ( $-\mathrm{C}$ strains, data not shown). Interestingly, all D-Phg expression samples contained higher amounts of Phg than the respective L-Phg expression samples (Fig. 3). Altogether, from all tested strains, S. lividans and S. pristinaespiralis turned out to be the optimal hosts for fermentative Phg production.

\section{Optimal production media for Phg production}

In order to define the best Phg production conditions, the optimal producer strains S. pristinaespiralis (SPlpg-OE, SPdpg-OE) and S. lividans (SLlpg-OE, SLdpg-OE) were grown in two different culture media - the complex medium R5 and the pristinamycin production medium HT7T. Samples were taken at different time points $(24,48,72$, and $96 \mathrm{~h})$ and Phg amount was determined by HPLC-MS/MS. Phg was detected in all $S$. pristinaespiralis (SPlpg-OE, SPdpg-OE) and S. lividans (SLlpg-OE, SLdpg-OE) expression samples, whereas only trace amounts of Phg were measured in the respective pRM4 control samples (Fig. 4a-d). Overall, Phg production was generally higher (even if statistically significant only for L-Phg expression samples as shown in Fig. S2) and more consistent in HT7T medium than in R5 (Fig. 4b, d vs a, c). Interestingly, Phg concentrations decreased in nearly all media and all expression hosts after reaching the maximal level, which suggests a degradation or metabolization of the expression product. An exception was found for $S$. lividans expression strains in HT7T medium, where Phg production steadily
Phg production in different host strains

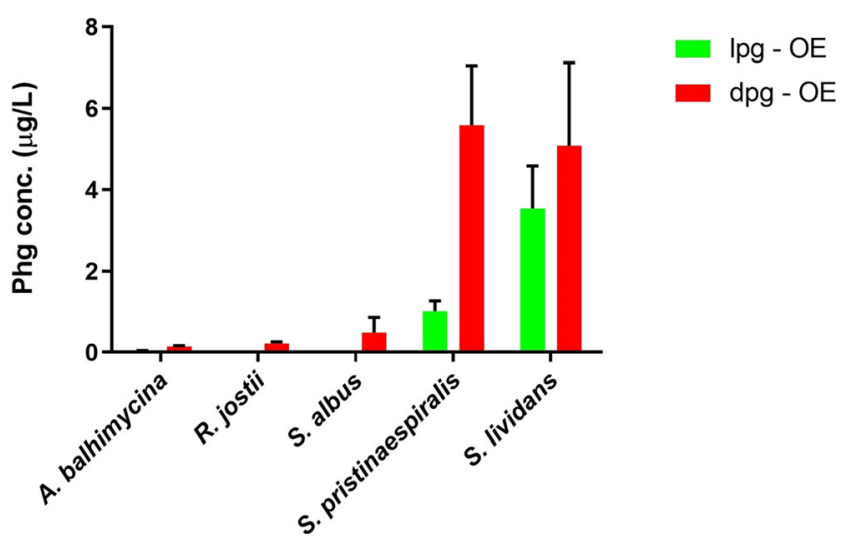

Fig. 3 Phg production of the different expression strains A. balhimycina (ABlpg-OE, ABdpg-OE), R. jostii (RJlpg-OE, RJdpg-OE), S. albus (SAlpg-OE, SAdpg-OE), S. pristinaespiralis (SPlpg-OE, SPdpg-OE), and S. lividans (SLlpg-OE, SLdpg-OE) grown in R5 medium. Phg production was measured at $30 \mathrm{~h}$. Phg concentration is given in micrograms per liter. Data were obtained from three independent biological replicates

increased to cultivation time point 96 h (Fig. 4b). Thus, Phg metabolization in $S$. lividans seems to be medium dependent. For S. pristinaespiralis samples, Phg decrease in the pristinamycin production medium HT7T might also be explained by a subsequent incorporation of Phg into PI. Furthermore, it was observed that Phg concentrations in general were higher in D-Phg expression strains than in L-Phg expression strains, which was consistent with the data obtained from the Phg expression studies in different host strains (Fig. 4a-d vs Fig. 3). D-amino acids are known for their poor metabolic usability (Elmadfa and Leitzmann 2015). Hence, the higher Phg amount in the D-Phg expression strains might be explained by a rather poor metabolization of the unnatural D-Phg enantiomer. Due to the observation that overall Phg production was more stable and consistent in HT7T and with regard to subsequent genetic engineering approaches targeting pristinamycin-specific genes in $S$. pristinaespiralis host strains (see below), the pristinamycin production medium HT7T was used as Phg production medium for further analyses.

\section{Deletion of a gene of the phenylacetyl-CoA degradation pathway significantly improves $\mathrm{Phg}$ production in S. pristinaespiralis but not in S. lividans}

In order to increase Phg production in the optimal producer strains S. lividans and S. pristinaespiralis, we aimed to genetically manipulate key steps within primary metabolism involved in precursor supply to direct the metabolic flux towards Phg production. As a target of manipulation, we chose the phenylacetyl-CoA degradation pathway since phenylacetyl$\mathrm{CoA}$ is a suggested precursor for the biosynthesis of Phg (Mast et al. 2011a; Osipenkov et al. 2018) (Fig. 2). In a previous study from Zhao et al. (2015), it has been reported that the 
a)

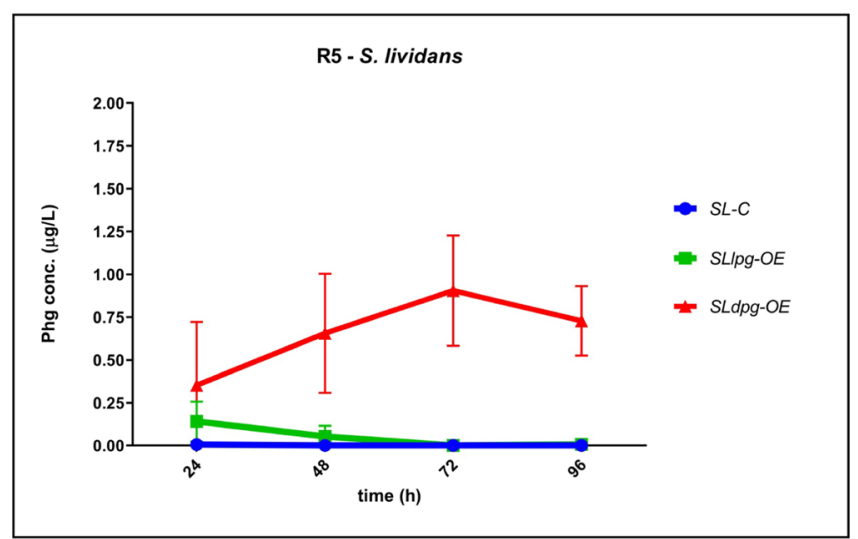

c)

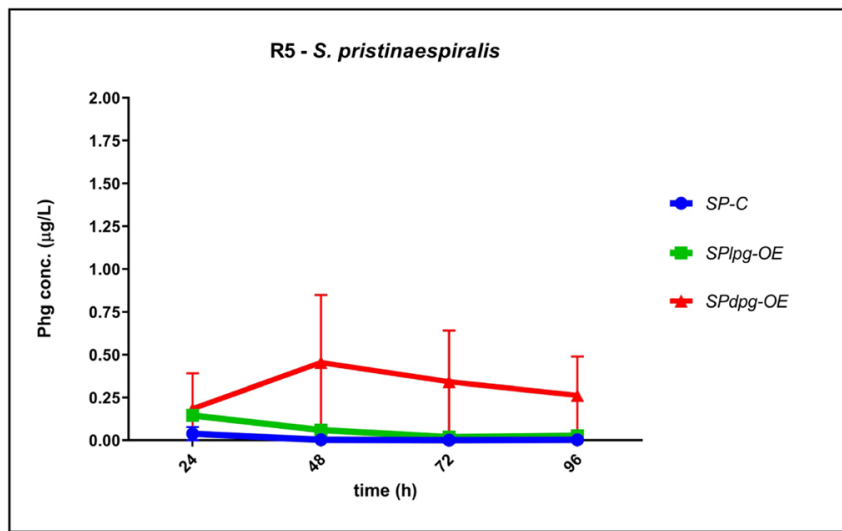

Fig. 4 Phg production of S. lividans Phg expression strains SLlpg-OE and $S L d p g-O E$, (control: $S L-C$ ) in R5 (a) and HT7T (b). Phg production of S. pristinaespiralis $\mathrm{Phg}$ expression strains SPlpg-OE and SPdpg-OE,

paaABCDE (paa) operon from S. pristinaespiralis encodes a putative phenylacetyl-CoA epoxidase multicomponent enzyme system, which is responsible for the degradation of phenylacetyl-CoA (Zhao et al. 2015). It was suggested that derepression of the paa operon in S. pristinaespiralis leads to a higher flux of phenylacetyl-CoA towards the phenylacetic acid catabolic pathway and thus to less precursor supply for L-Phg biosynthesis (Zhao et al. 2015). By contrast, it can be assumed that an inactivation of the paa genes in S. pristinaespiralis drives the phenylacetyl-CoA flux towards Phg biosynthesis. Thus, we aimed to inactivate the paa operon in S. pristinaespiralis - but also S. lividans, since a homologous paa operon is present in the $S$. lividans genome (Supplementary File) - and overexpress the Phg operons in the engineered mutant strains in order to increase production yields. For this purpose, the gene region paaA-E in $S$. pristinaespiralis and $S$. lividans, respectively, was inactivated by replacing it against a thiostrepton resistance cassette $\left(\right.$ thio $\left.^{R}\right)$ (Supplementary File, Fig. S3). This resulted in the mutants SPpaa::thio and SLpaa: thio, respectively, in which the Phg expression constructs $\mathrm{pYM} / \mathrm{lpg}$ and $\mathrm{pYM} / \mathrm{dpg}$, as well as the empty vector as b)

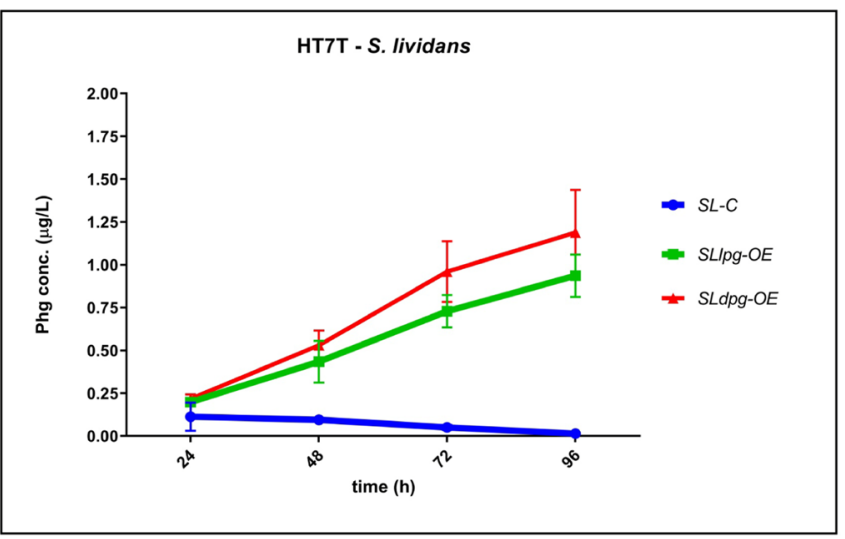

d)

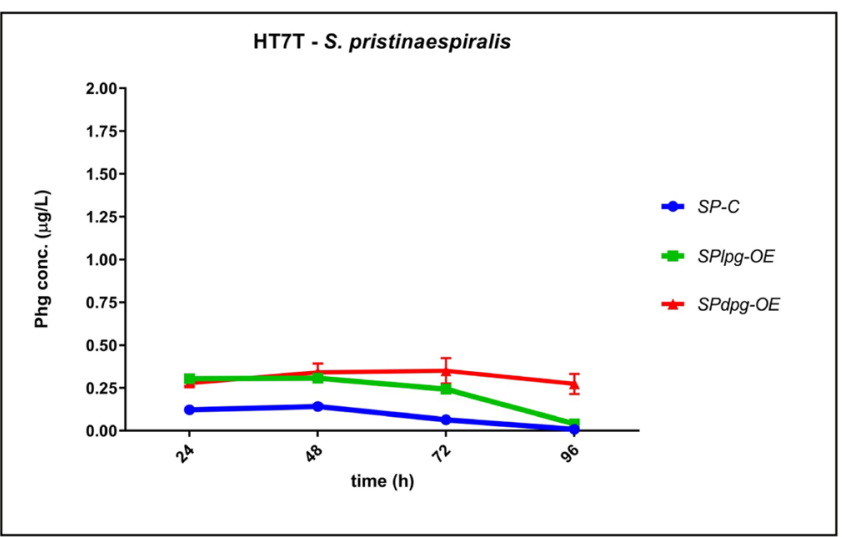

(control: $S P-C$ ) in R5 (c) and HT7T (d). Phg production was measured at $24,48,72$, and $96 \mathrm{~h}$. Phg concentration is given in micrograms per liter. Data were obtained from three independent biological replicates

a control, were each transferred to. The paa control strains, SPpaa::thio-C and SLpaa::thio-C and the host strains SPpaa::thio lpg-OE, SPpaa::thio dpg-OE, SLpaa::thio lpg$O E$, and SLpaa::thio dpg-OE were grown in HT7T medium and supernatant samples at different time points were used for Phg production analysis. HPLC-MS/MS measurements of the samples from the engineered host strains revealed that Phg production in the $S$. lividans paa expression samples was almost the same as in the wild-type-derived expression samples (Fig. 5a vs Fig. 4b): maximal Phg production at $96 \mathrm{~h}$ was measured for SLpaa: :thio $l p g-O E$ at $1.00 \mu \mathrm{g} / \mathrm{L}$ compared with $0.94 \mu \mathrm{g} / \mathrm{L}$ for SLlpg-OE and $0.95 \mu \mathrm{g} / \mathrm{L}$ for SLpaa: :thio dpg-OE compared with $1.2 \mu \mathrm{g} / \mathrm{L}$ for SLdpg-OE. In contrast, Phg production was strongly improved for $S$. pristinaespiralis paa-derived expression samples (Fig. 5b vs Fig. 4d): Already after $24 \mathrm{~h}$, Phg amount in SPpaa::thio lpg-OE $(1.57 \mu \mathrm{g} / \mathrm{L})$ was 5fold higher than in SPlpg-OE $(0.31 \mu \mathrm{g} / \mathrm{L})$ and remained high until $96 \mathrm{~h}$. Here, the production decline at $72 \mathrm{~h}$ might be an artifact since standard deviations for the SPpaa::thio lpg-OE samples in general were quite high. Phg production was also significantly improved for SPpaa::thio dpg-OE strains, where 
the maximal Phg production at $96 \mathrm{~h}(1.30 \mu \mathrm{g} / \mathrm{L})$ was 3.7-fold higher than in non-engineered SPdpg-OE strains $(0.35 \mu \mathrm{g} / \mathrm{L})$. Overall, the significant improvement of Phg production in S. pristinaespiralis paa host strains most likely results from the directed flux of the phenylacetyl-CoA precursor towards the Phg biosynthetic pathway. The fact that Phg production was improved for SPpaa::thio lpg-OE compared with SPpaa::thio dpg-OE might be explained by the different enzyme kinetics of the two aminotransferases. D-amino acid transaminases, such as HpgAT (encoded in the dpg operon), are commonly known to have a very low transamination activity towards D-Phg (Soda and Esaki 1994). Thus, PglE may convert the accruing phenylglyoxylate precursor more efficiently to L-Phg than HpgAT can convert it to D-Phg.

\section{Deletion of Phg aminotransferase gene pgIE slightly improves Phg production in S. pristinaespiralis}

In a recent study, we showed that the L-Phg aminotransferase $\mathrm{PglE}$ is responsible for the conversion of phenylglyoxylate to LPhg in S. pristinaespiralis (Osipenkov et al. 2018) (Fig. 2). Deletion of $\mathrm{pglE}$ leads to an accumulation of phenylglyoxylate (Osipenkov et al. 2018). Due to this increased basal precursor availability, we were interested how the Phg operon expression in the $S$. pristinaespiralis pglE mutant ( $M p g l E$ ) would influence production performance. Besides that, inactivation of the native $p g l E$ gene could deliver a genetic background for the production of enantiopure Phgs in S. pristinaespiralis. Thus, the $M p g l E$ mutant was used as parental strain for the expression of the Phg operons. Strain denomination is similar as reported above and samples were treated as outlined before. HPLC-MS/ MS analysis revealed that $\mathrm{Phg}$ production in $\mathrm{MpglE}$ host strains (MpglE lpg-OE and MpglE dpg-OE) was overall slightly higher than in S. pristinaespiralis wild-type-derived strains (Fig. 6 vs Fig. 4d): An improvement was observed for the MpglE lpg-OE

a)

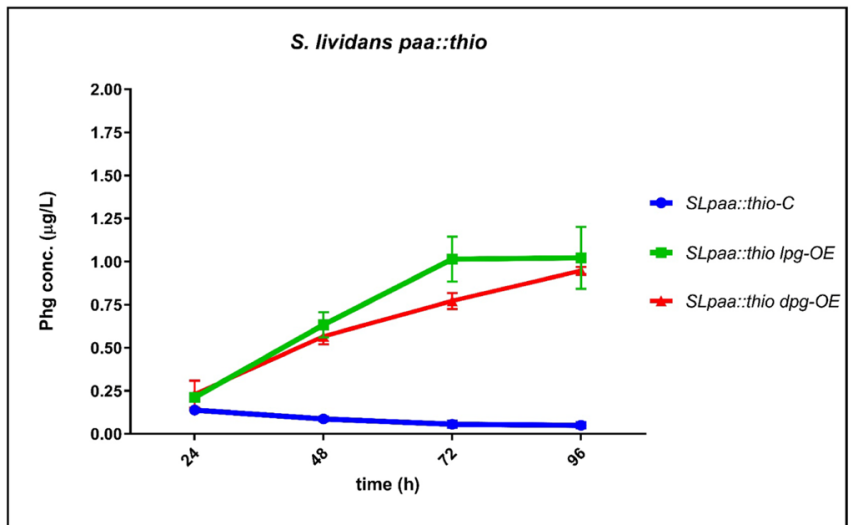

Fig. 5 Phg production of S. lividans paa host strains SLpaa::thio lpg-OE and SLpaa::thio dpg-OE, (control: SLpaa::thio-C) (a) and $S$. pristinaespiralis paa host strains SPpaa::thio lpg-OE and SPpaa::thio dpg-OE, (control: SPpaa::thio-C) (b) grown in HT7T. Phg samples, where a maximal production of $0.56 \mu \mathrm{g} / \mathrm{L}$ Phg at $48 \mathrm{~h}$ was measured, which is an increase of 1.8 -fold compared with the maximal value of $0.31 \mu \mathrm{g} / \mathrm{L}$ Phg at $24 \mathrm{~h}$ in the SPlpg-OE sample. For MpglE dpg-OE expression samples, no tremendous Phg production improvement was observed (Fig. 6). Therefore, one could speculate that the slightly increased $\mathrm{Phg}$ rates in $\mathrm{MpglE}$ lpg-OE may result from a somehow favorable basal phenylglyoxylate precursor supply.

\section{Deletion of the PI-NRPS gene snbDE significantly improves Phg production in S. pristinaespiralis}

As suggested above, the decrease of Phg in SPlpg-OE samples may be due to an incorporation of L-Phg into PI (Fig. 4d). Thus, a strategy to increase Phg production in S. pristinaespiralis is to block PI biosynthesis. In order to do that, we inactivated the gene $s n b D E$ in S. pristinaespiralis (Supplementary File), which encodes the PI-specific NRPS module SnbDE that uses L-Phg as a building block for PI biosynthesis (Mast et al. 2011b). The respective mutant MsnbDE::thio was used as expression host for the Phg operon expression. The derived host strains MsnbDE::thio lpg-OE and MsnbDE::thio dpg-OE, as well as the control MsnbDE::thio-C, were grown in HT7T and samples were analyzed for Phg production by HPLC-MS/MS. HPLCMS/MS analysis revealed a maximal Phg production in samples MsnbDE::thio lpg-OE $(0.87 \mu \mathrm{g} / \mathrm{L})$ and MsnbDE::thio $d p g-O E(1.27 \mu \mathrm{g} / \mathrm{L})$ at $96 \mathrm{~h}$, which was an increase of $\sim 3$-fold compared with maximal production values in wild-typederived samples SPlpg-OE and SPdpg-OE $(0.30 \mu \mathrm{g} / \mathrm{L}$ and $0.35 \mu \mathrm{g} / \mathrm{L}$ ), respectively (Fig. 7 vs Fig. 4d). Furthermore, it was found that Phg concentration in the MsnbDE::thio-derived strains increased continuously, whereas a decrease was observed in the wild-type-derived samples at later time points. Actually, the Phg production profile of the MsnbDE::thio-derived strains more resembled the production profile of the

b)

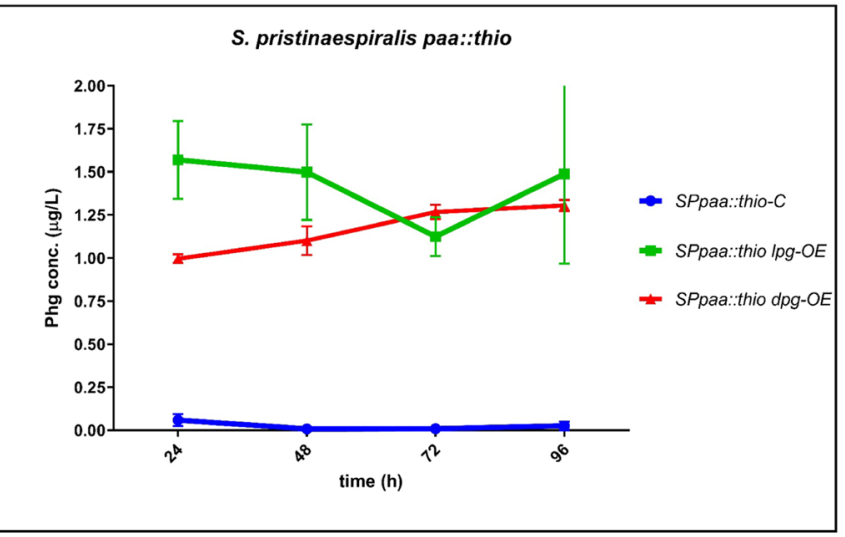

production was measured at $24,48,72$, and $96 \mathrm{~h}$. Phg concentration is given in micrograms per liter. Data were obtained from three independent biological replicates 
Fig. 6 Phg production of $S$. pristinaespiralis MpglE host strains MpglE lpg-OE and MpglE dpg-OE, (control: $M p g l E-C$ ) grown in HT7T. Phg production was measured at 24, 48, 72, and $96 \mathrm{~h}$. Phg concentration is given in micrograms per liter. Data were obtained from three independent biological replicates

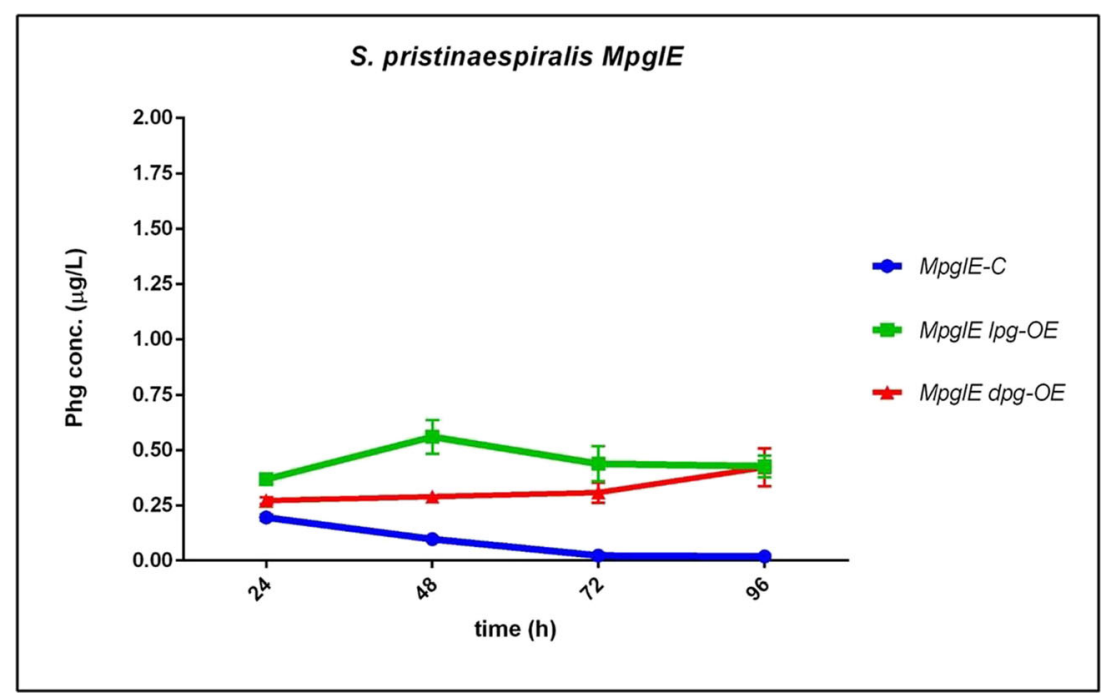

S. lividans host strains (Fig. 7 vs Fig. 4b). Thus, it can be assumed that Phg production in the MsnbDE::thio-derived strains is steadily increasing because Phg is not utilized for PI biosynthesis and thus accumulates, which may also happen in S. lividans because this strain does not produce pristinamycin.

\section{Deletion of the pristinamycin TetR-like regulatory gene papR5 significantly improves Phg production in S. pristinaespiralis}

As we had incident that Phg production performance in $S$. pristinaespiralis depends on the pristinamycin biosynthesis capability (see above for $M p g l E, M s n b D E$ samples), we aimed to further enhance Phg production by using a pristinamycin superproducer as expression host. In a previous study, we showed that the $S$. pristinaespiralis repressor mutant papR5::apra produces up to $\sim 300 \%$ more pristinamycin than the wild-type strain (Mast et al. 2015). Due to this high pristinamycin production capability, we used papR5::apra as a host for Phg operon expression. The derived host strains papR5::apra lpg-OE and papR5::apra dpg-OE, as well as the control strain papR5::apra-C, were grown in HT7T and samples were analyzed by HPLC-MS/MS for Phg production. HPLC-MS/MS data revealed that Phg production was significantly increased in papR5::apra-derived host strains compared with the wild-type-derived ones: papR5::apra lpg-OE and papR5::apra dpg-OE produced approximately 3.3-fold and 2-fold, respectively, more Phg than the wild-typederived expression strains (papR5::apra lpg-OE: $1 \mu \mathrm{g} / \mathrm{L}$; papR5::apra dpg-OE: $0.72 \mu \mathrm{g} / \mathrm{L} \mathrm{Phg)} \mathrm{(Fig.} 8$ vs Fig. $4 \mathrm{~d}$ ). Phg production was increased especially in the papR5::apra lpg-OE host strain, which was also observed for the other Phg precursor-engineered host strains (SLpaa::thio, SPpaa::thio, and $M p g l E)$. Thus, Phg-related precursor engineering seems to affect more L-Phg than D-Phg biosynthesis. This might be explained by the less favorable enzymatic properties of HpgAT, as
Fig. 7 Phg production of S. pristinaespiralis snbDE host strains MsnbDE: :thio lpg-OE and MsnbDE::thio dpg-OE, (control: $M s n b D E:$ :thio-C) grown in HT7T. Phg production was measured at 24, 48, 72, and $96 \mathrm{~h}$. Phg concentration is given in micrograms per liter. Data were obtained from three independent biological replicates

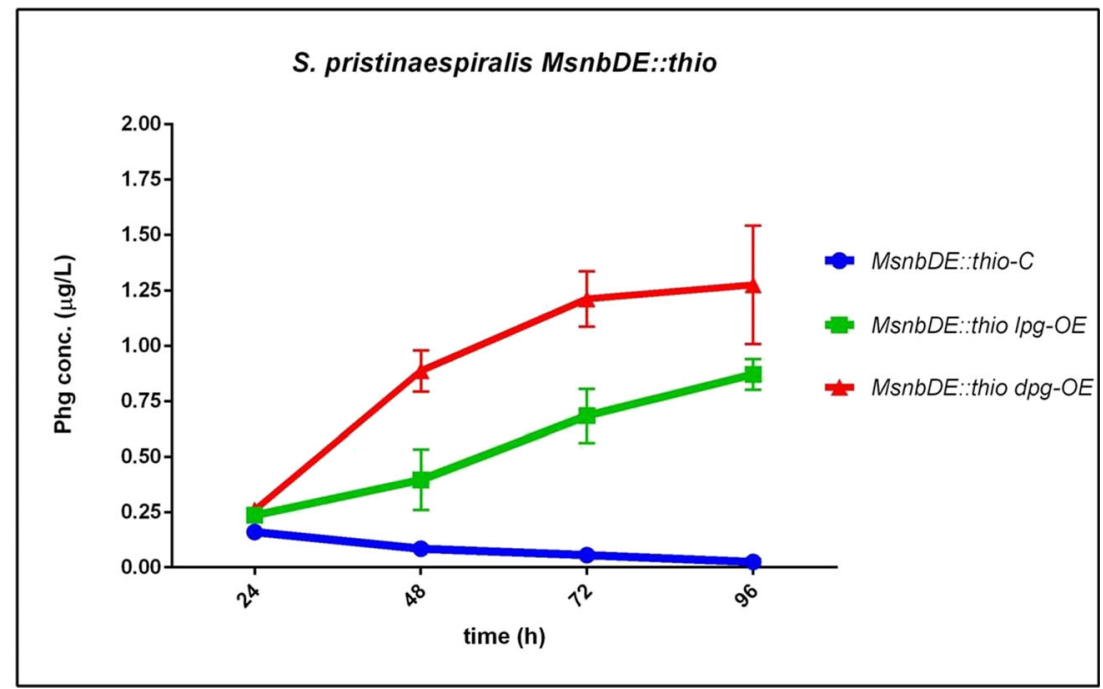


Fig. 8 Phg production in the $S$. pristinaespiralis papR5 host strains papR5::apra lpg-OE and papR5::apra dpg-OE, (control: papR5::apra-C) grown in HT7T. Phg production was measured at 24, 48, 72, and $96 \mathrm{~h}$. Phg concentration is given in micrograms per liter. Data were obtained from three independent biological replicates

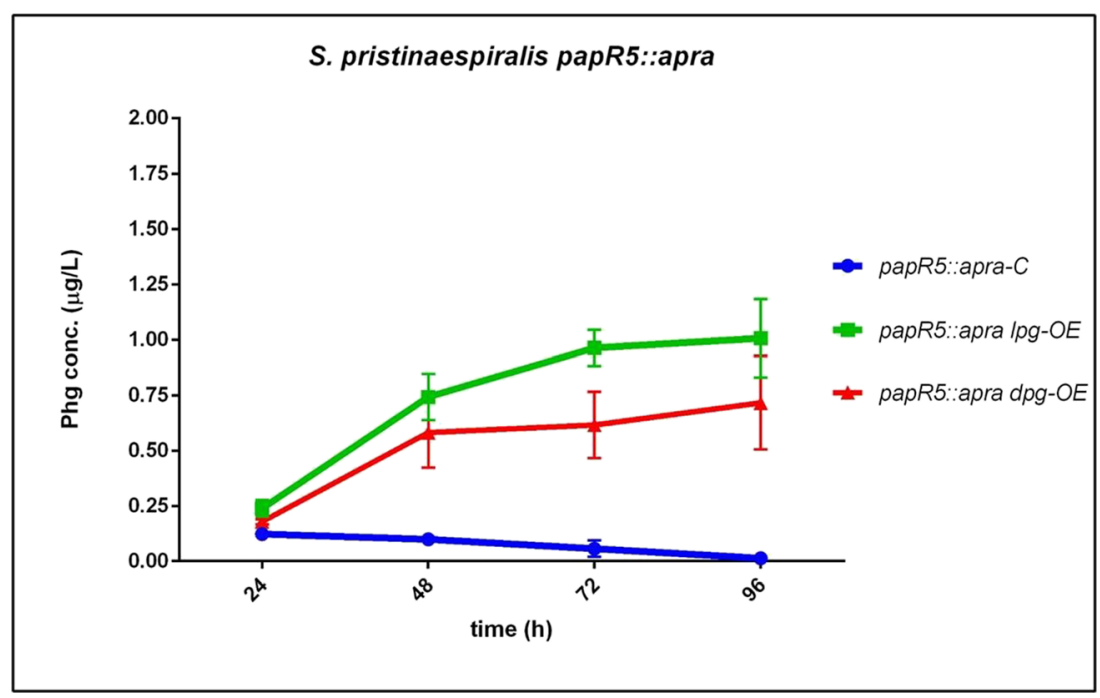

mentioned before. The reason why Phg concentration is stable or even increasing in these expression hosts might be because PI production is oversaturated with Phg precursor and thus Phg would accumulate. Overall, the improvement of Phg production in the papR5::apra-derived host strains most likely results from the elevated levels of precursor supply in the course of an increased pristinamycin biosynthesis.

\section{Discussion}

Non-proteinogenic amino acids, such as Phg, are important building blocks and precursors for the synthesis of industrial relevant pharmaceuticals and other fine chemicals. We could show that genetic engineering of suitable target genes in appropriate expression strains allows improvement of Phg production. As expression hosts, we tested different actinomycetal species. This is the first time that Phg production has been accomplished in actinomycetes. Even if Phg production was quite efficient in $S$. lividans expression strains, $S$. pristinaespiralis is preferred as producer host since it offers a broader range of target genes, suitable for genetic engineering to increase production yields. The strongest Phg production improvement was observed for S. pristinaespiralis expression strains with an inactivated phenylacetyl-CoA degradation pathway (SPpaa::thio strains). Here, production levels were quite high from the beginning on, which might be the result of so far unknown feedback/ feedforward control of Phg biosynthesis in S. pristinaespiralis. Maximal production of SPpaa::thio was measured at $\sim 1.6 \mu \mathrm{g} / \mathrm{L}$ Phg, which corresponds to a 4.6-fold increase compared with the production levels of wild-type-derived strains. However, also mutations of specific PI biosynthesis genes ( $p g l E$, snbDE) and a pristinamycin transcriptional regulator gene (papR5) led to an increase of Phg production. Especially for these producer strains it would be interesting to also determine PI production levels in order analyze how they correlate with Phg production profiles. Furthermore, it should be investigated if D-Phg can be used as a building block for PI biosynthesis, which is not known so far. This could be assumed since Phg production was increased in both PI-NRPS deletion host strains, MsnbDE::thio lpg-OE and MsnbDE::thio dpg-OE, suggesting that both Phg enantiomers are used as PI building blocks.

A logic strategy to further increase Phg production is to perform a combinatory genetic engineering approach and inactivate all the above-mentioned genes in S. pristinaespiralis. An additional target gene for further production improvement is papR2, which encodes a SARP-type transcriptional regulator that is suggested to activate the L-Phg operon in S. pristinaespiralis (Mast et al. 2015). Thus, additional overexpression of papR2 in an engineered S. pristinaespiralis Phg superhost could further increase production yields. Due to these multifarious manipulation opportunities, S. pristinaespiralis indeed represents a good chassis strain for fermentative Phg production. However, even if production improvement worked out quite well for $S$. pristinaespiralis, the overall product concentrations are still low. Maximal production was $\sim 1.6 \mu \mathrm{g} / \mathrm{L} \mathrm{Phg,}$ which is still far away from mg production levels previously reported for E. coli fermentation (Müller et al. 2006).

In $E$. coli, Phg production was accomplished by expressing an artificial Phg operon, consisting of at least three genes (hmaS, hmo, hpgAT, or pgat) from different organisms (Streptomyces coelicolor, Amycolatopsis orientalis, and Pseudomonas putida) in a suitable pathway-engineered E. coli strain (Müller et al. 2006; Liu et al. 2014). Thereby, Phg production was optimized to $51.6 \mathrm{mg} / \mathrm{g}$ dry cell weight LPhg (Liu et al. 2014, 2015) and 102 mg/g dry cell weight DPhg (Müller et al. 2006). In another approach, L-Phg production was accomplished in $E$. coli by co-expression of a leucine dehydrogenase from Bacillus cereus $(B c \mathrm{LeuDH})$ and a $\mathrm{NAD}^{+}$-dependent mutant formate dehydrogenase from Candida boidinii $\left(\mathrm{CbFDH}_{\mathrm{A} 10 \mathrm{C}}\right)$, which yielded $28.4 \mathrm{mg} / \mathrm{g}$ dry cell weight L-Phg (Liu et al. 2018). However, these values 
are not comparable with those of our study since production volumes are given in $\mathrm{mg} / \mathrm{g}$ biomass in the $E$. coli studies but were measured in micrograms per liter of Phg from culture supernatant samples in the present study. Indeed, it was not possible to correlate biomass values (dry cell weight) with Phg production outputs in Streptomyces samples due to their irregular growth in liquid cultures, which leads to strong deviations in biomass values. In any way, absolute Phg concentrations for Streptomyces samples are hard to determine exactly by HPLCMS/MS quantification. What we rather would like to depict is that Phg production and optimization can clearly be followed by comparison with control strains. Notably, this is the first time that fermentative Phg production has been reported for actinomycetes. Furthermore, it is important to mention that, unlike the studies in E. coli, the precursor supply of phenylpyruvate was not modified in our strains. This is a pivotal target for further studies as usually the aromatic amino acid biosynthesis pathway in bacteria is strictly regulated and limits the precursor supply (Huccetogullari et al. 2019; Lee and Wendisch 2017; Rodriguez et al. 2014; Sprenger 2007). It was shown for Streptomyces venezuelae that an improved flux through the shikimate pathway by overexpressing the genes of shikimate kinase (aroK) and dehydroquinate synthase $(a r o B)$ increased the production of the aromatic antibiotic chloramphenicol (Vitayakritsirikul et al. 2016). Overexpression of the gene chorismate synthase $($ aroC) in Streptomyces tsukubaensis improved the production of the immunosuppressant tacrolimus (Wang et al. 2017). Furthermore, overexpression of the 3-deoxy-D-arabinoheptulosonate 7-phosphate synthase gene (dahp) and the prephenate dehydrogenase gene $(p d h)$ in A. balhimycina resulted in improved balhimycin production (Thykaer et al. 2010). Thus, it is very likely that Phg amount can be further increased by improving precursor supply from the shikimate pathway. In our study, we could ensure and show that the final reaction, the conversion of phenylpyruvate to $\mathrm{Phg}$, can be improved by deleting the competing reaction for Phg biosynthesis.

S. pristinaespiralis-derived Phg operons indeed are interesting molecular entities for future applications. So far, the $p g l$ genes from $S$. pristinaespiralis encode the only known natural Phg biosynthesis pathway. One could assume that this pathway is already evolutionary optimized and under appropriate conditions might be more powerful than an artificially assembled pathway harboring genes from different origins. However, this would require further investigations. Overall, the Phg operons represent promising biobricks for Phg-related production processes. A purely fermentative production route has mainly been prevented by the absence of a natural Phg pathway. In this study, we could describe the functionality of the natural L-Phg operon from S. pristinaespiralis and its derived D-Phg operon obtained by a synthetic biology approach. The new fermentative Phg production route serves as a basis to replace the environmentally unfriendly industrial Phg production process.
Authors' contributions DM, VM, NO, and SK performed experiments; ROW, FH, OH, and JWY contributed to experiments; GS contributed to data analysis, AK carried out HPLC-MS/MS analysis; YM designed, supervised, and coordinated the study. YM wrote the manuscript. $\mathrm{OH}$ contributed to manuscript editing. All authors read and approved the final manuscript.

Funding information Open Access funding provided by Projekt DEAL. This work was supported by the Institutional Strategy of the University of Tübingen (Deutsche Forschungsgemeinschaft, ZUK 63). We further received funding from the Baden-Württemberg Stiftung (BWST_WSF035) and the German Center for Infection research (DZIF) (TTU 09.819).

\section{Compliance with ethical standards}

Conflict of interest The authors declare that they have no competing interests.

Ethical approval This article does not contain any studies with human participants or animals performed by any of the authors.

Open Access This article is licensed under a Creative Commons Attribution 4.0 International License, which permits use, sharing, adaptation, distribution and reproduction in any medium or format, as long as you give appropriate credit to the original author(s) and the source, provide a link to the Creative Commons licence, and indicate if changes were made. The images or other third party material in this article are included in the article's Creative Commons licence, unless indicated otherwise in a credit line to the material. If material is not included in the article's Creative Commons licence and your intended use is not permitted by statutory regulation or exceeds the permitted use, you will need to obtain permission directly from the copyright holder. To view a copy of this licence, visit http://creativecommons.org/licenses/by/4.0/.

\section{References}

Al Toma RS, Brieke C, Cryle MJ, Süssmuth RD (2015) Structural aspects of phenylglycines, their biosynthesis and occurrence in peptide natural products. Nat Prod Rep 32:1207-1235

Barredo JL (2005) Methods in biotechnology: microbial enzymes and biotransformations. Humana Press, Totowa ISSN 1940-6061

Croteau R, Ketchum REB, Long RM, Kaspera R, Wildung MR (2006) Taxol biosynthesis and molecular genetics. Phytochem Rev 5:75-97

Denis JN, Correa A, Greene AE (1991) Direct, highly efficient synthesis from (S)-(+)-phenylglycine of the taxol and taxotere side chains. J Org Chem 56:6939-6942

Ebeling SC (1998) The synthesis of artificial sweeteners (phenylglycineanalogues of aspartame) in order to evaluate changes in the $\gamma$ glycophore component. Food Chem 61:107-112

Elmadfa I, Leitzmann C (2015) Ernährung des Menschen, 5th edn. Eugen Ulmer KG, Stuttgart

Folcher M, Gaillard H, Nguyen LT, Nguyen KT, Lacroix P, BamasJacques N, Rinkel M, Thompson CJ (2001) Pleiotropic functions of a Streptomyces pristinaespiralis autoregulator receptor in development, antibiotic biosynthesis, and expression of a superoxide dismutase. J Biol Chem 276:44297-44306

Global Industry Analysts Inc (2016) A worldwide business strategy \& market intelligence source. MCP-7644: D-Amino Acids - A Global Strategic Business Report. http://www.strategyr.com/PressMCP7644.asp. Accessed June 13, 2019

Huccetogullari D, Lou ZW, Lee SY (2019) Metabolic engineering of microorganisms for production of aromatic compounds. Microb Cell Factories 18:41 
Janusz JM (1986) Alpha-L-aspartyl-D-phenylglycine esters and amides useful as high intesity sweeteners. European patent EP0168112A2

Kan T, Tominari Y, Rikimaru K, Morohashi Y, Natsugari H, Tomita T, Iwatsubo T, Fukuyama T (2004) Parallel synthesis of DAPT derivatives and their gamma-secretase-inhibitory activity. Bioorg Med Chem Lett 14:1983-1985

Kieser T, Bibb MJ, Buttner MJ, Chater KF, Hopwood DA (2000) Practical Streptomyces genetics. John Innes Foundation, Norwich

Lee JH, Wendisch VF (2017) Biotechnological production of aromatic compounds of the extended shikimate pathway from renewable biomass. J Biotechnol 257:211-221

Liu SP, Liu RX, El-Rotail AAMM, Ding ZY, Gu ZH, Zhang L, Shi GY (2014) Heterologous pathway for the production of L-phenylglycine from glucose by E. coli. J Biotechnol 186:91-97

Liu SP, Zhang L, Mao J, Ding ZY, Shi GY (2015) Metabolic engineering of Escherichia coli for the production of phenylpyruvate derivatives. Metab Eng 32:55-65

Liu Q, Zhou J, Yang T, Zhang X, Xu M, Rao Z (2018) Efficient biosynthesis of L-phenylglycine by an engineered Escherichia coli with a tunable multi-enzyme-coordinate expression system. Appl Microbiol Biotechnol 102:2129-2141

$\mathrm{Lu} \mathrm{Y,} \mathrm{Freeland} \mathrm{S} \mathrm{(2006)} \mathrm{On} \mathrm{the} \mathrm{evolution} \mathrm{of} \mathrm{the} \mathrm{standard} \mathrm{amino-acid}$ alphabet. Genome Biol 7:102

Mast Y, Wohlleben W (2014) Streptogramins - two are better than one! Int J Med Microbiol 304:44-50

Mast Y, Wohlleben W, Schinko E (2011a) Identification and functional characterization of phenylglycine biosynthetic genes involved in pristinamycin biosynthesis in Streptomyces pristinaespiralis. J Biotechnol 155:63-67

Mast Y, Weber T, Gölz M, Ort-Winklbauer R, Gondran A, Wohlleben W, Schinko E (2011b) Characterization of the "pristinamycin supercluster" of Streptomyces pristinaespiralis. Microb Biotechnol 4:192-206

Mast Y, Guezguez J, Handel F, Schinko E (2015) A complex signaling cascade governs pristinamycin biosynthesis in Streptomyces pristinaespiralis. Appl Environ Microbiol 81:6621-6636

Müller U, van Assema F, Gunsior M, Orf S, Kremer S, Schipper D, Wagemans A, Townsend CA, Sonke T, Bovenberg R, Wubbolts M (2006) Metabolic engineering of the E. coli L-phenylalanine pathway for the production of D-phenylglycine (D-Phg). Metab Eng 8:196-208

Müller UM, Boer R, Bovenberg R (2013) MbtH-like proteins in the production of semi-synthetic antibiotics. Patent WO2013113646A1

Nah H-J, Pyeon H-R, Kang S-H, Choi S-S, Kim E-S (2017) Cloning and heterologous expression of a large-sized natural product biosynthetic gene cluster in Streptomyces species. Front Microbiol 8:394

Ningsih F, Kitani S, Fukushima E, Nihira T (2011) VisG is essential for biosynthesis of virginiamycin $\mathrm{S}$, a streptogramin type B antibiotic, as a provider of the nonproteinogenic amino acid phenylglycine. Microbiology 157:3213-3220

Osipenkov N (2016) Biosyntheseweg eines natürlichen Phenylglycins, Biochemische Analyse und Perspektiven einer nachhaltigen Produktion. Springer Fachmedien, Wiesbaden ISBN: 9783658118648

Osipenkov N, Kulik A, Mast Y (2018) Characterization of the phenylglycine aminotransferase PglE from Streptomyces pristinaespiralis. J Biotechnol 278:34-38

Pfeifer V, Nicholson GJ, Ries J, Recktenwald J, Schefer AB, Shawky RM, Schröder J, Wohlleben W, Pelzer S (2001) A polyketide synthase in glycopeptide biosynthesis. The biosynthesis of the nonproteinogenic amino acid (S)-3,5-dihydroxyphenylglycine. J Biol Chem 276:38370-38377

Pridmore RD (1987) New and versatile cloning vectors with kanamycinresistance marker. Gene 56:309-312
Rodriguez A, Martnez J, Flores N, Escalante A, Gosset G, Bolivar F (2014) Engineering Escherichia coli to overproduce aromatic amino acids and derived compounds. Microb Cell Factories 13:126

Sambrook J, Fritsch EF, Maniatis T (1989) Molecular cloning: a laboratory manual, 2nd edn. Cold Spring Harbor laboratory press, New York

Schmid A, Dordick JS, Hauer B, Kiener A, Wubbolts M, Witholt B (2001) Industrial biocatalysis today and tomorrow. Nature 11:258-268

Schutt H (1981) Stereoselective resolution of phenylglycine derivatives and 4-hydroxyphenylglycine derivatives with enzyme resins. US patent US4260684A

Shiau CY, Pai SC, Lin WP, Ji DD, Liu YT (2005) Purification and characterization of inducible cephalexin synthesizing enzyme in Gluconobacter oxydans. Biosci Biotechnol Biochem 69:463-469

Soda K, Esaki N (1994) Pyridoxal enzymes acting on D-amino acids. Pure Appl Chem 66:709-714

Sprenger GA (2007) From scratch to value: engineering Escherichia coli wild type cells to the production of L-phenylalanine and other fine chemicals derived from chorismate. Appl Microbiol Biotechnol 75:739-749

Stevenazzi A, Marchini M, Sandrone G, Vergani B, Lattanzio M (2014) Amino acidic scaffolds bearing unnatural side chains: an old idea generates new and versatile tools for the life sciences. Bioorg Med Chem Lett 24:5349-5356

Thykaer J, Nielsen J, Wohlleben W, Weber T, Gutknecht M, Lantz AE, Stegmann E (2010) Increased glycopeptide production after overexpression of shikimate pathway genes being part of the balhimycin biosynthetic gene cluster. Metab Eng 12:455-461

Vedha-Peters K, Gunawardana M, Rozzell JD, Novick SJ (2006) Creation of a broad-range and highly stereoselective D-amino acid dehydrogenase for the one-step synthesis of D-amino acids. J Am Chem Soc 128:10923-10929

Vitayakritsirikul V, Jaemsaeng R, Lohmaneeratana K, Thanapipatsiri A, Daduang R, Chuawong P, Thamchaipenet A (2016) Improvement of chloramphenicol production in Streptomyces venezuelae ATCC 10712 by overexpression of the $\operatorname{aro} B$ and aroK genes catalysing steps in the shikimate pathway. Antonie Van Leeuwenhoek 109:379-388

Walsh CT, O’Brien RV, Khosla C (2013) Nonproteinogenic amino acid building blocks for nonribosomal peptide and hybrid polyketide scaffolds. Angew Chem Int Ed 52:7098-7124

Walton CJ, Thiebaut F, Brunzelle JS, Couture J, Chica RA (2018) Structural determinants of the stereo-inverting activity of Pseudomonas stutzeri D-phenylglycine aminotransferase. Biochemistry 57:5437-5446

Wang ZM, Kolb HC, Sharpless KB (1994) Large-scale and highly Enantioselective synthesis of the taxol C-13 side chain through asymmetric dihydroxylation. J Org Chem 59:5104-5105

Wang C, Liu J, Liu H, Liang S, Wen J (2017) Combining metabolomics and network analysis to improve tacrolimus production in Streptomyces tsukubaensis using different exogenous feedings. J Ind Microbiol Biotechnol 44:1527-1540

Wegman MA, Janssen MHA, van Rantwijk F, Sheldon RA (2001) Towards biocatalytic synthesis of beta-lactam antibiotics. Adv Synth Catal 343:559-576

Yam KC, Okamoto S, Roberts JN, Eltis LD (2011) Adventures in Rhodococcus- from steroids to explosives. Can J Microbiol 57: $155-168$

Zhao Y, Feng R, Zheng G, Tian J, Ruan L, Ge M, Jiang W, Lu Y (2015) Involvement of the TetR-type regulator PaaR in the regulation of pristinamycin I biosynthesis through an effect on precursor supply in Streptomyces pristinaespiralis. J Bacteriol 197:2062-2071

Publisher's note Springer Nature remains neutral with regard to jurisdictional claims in published maps and institutional affiliations. 